\title{
Effects of a novel ascorbate-based protocol on infarct size and ventricle function in acute myocardial infarction patients undergoing percutaneous coronary angioplasty
}

Cristóbal Ramos ${ }^{1}$, Roberto Brito ${ }^{2}$, Jaime González-Montero ${ }^{1,2}$, Nicolás Valls ${ }^{1,2}$, Juan G. Gormaz ${ }^{2}$, Juan C. Prieto ${ }^{1,2}$, Rubén Aguayo ${ }^{3}$, Ángel Puentes ${ }^{3}$, Viviana Noriega ${ }^{1}$, Gonzalo Pereira ${ }^{1}$, Tamara Palavecino ${ }^{1}$, Ramón Rodrigo ${ }^{2}$

${ }^{1}$ Faculty of Medicine, University of Chile, Santiago, Chile ${ }^{2}$ Molecular and Clinical Pharmacology Program, Institute of Biomedical Sciences, Faculty of Medicine, University of Chile, Santiago, Chile

${ }^{3}$ San Juan de Dios Hospital, Santiago, Chile

Submitted: 16 January 2016

Accepted: 15 March 2016

Arch Med Sci 2017; 13, 3: 558-567

DOI: 10.5114 /aoms.2016.59713

Copyright () 2016 Termedia \& Banach

\section{Abstract}

Introduction: This study was designed to test the hypothesis that high-dose ascorbate prior to reperfusion followed by low chronic oral doses ameliorate myocardial reperfusion injury (MRI) in acute myocardial infarction patients subjected to primary percutaneous coronary angioplasty (PCA).

Material and methods: A randomized double-blind placebo-controlled and multicenter clinical trial was performed on acute myocardial infarction (AMI) patients who underwent PCA. Sodium ascorbate $(320 \mathrm{mmol} / \mathrm{l}, n=53)$ or placebo $(n=46)$ was infused 30 min prior to PCA. Blood samples were drawn at enrolment (M1), after balloon deflation (M2), 6-8 h after M2 (M3) and at discharge (M4). Total antioxidant capacity of plasma (ferric reducing ability of plasma - FRAP), erythrocyte reduced glutathione (GSH) and plasma ascorbate levels were determined in blood samples. Cardiac magnetic resonance (CMR) was performed at 7-15 days and 2-3 months following PCA. Ninety-nine patients were enrolled. In 67 patients, the first CMR was performed, and 40 patients completed follow-up.

Results: The ascorbate group showed significantly higher ascorbate and FRAP levels and a decrease in the GSH levels at M2 and M3 $(p<0.05)$. There were no significant differences in the infarct size, indexed end-systolic volume and ejection fraction at both CMRs. There was a significant amelioration in the decreased ejection fraction between the first and second CMR in the ascorbate group $(p<0.05)$.

Conclusions: Ascorbate given prior to reperfusion did not show a significant difference in infarct size or ejection fraction. However, it improved the change in ejection fraction determined between 7-15 days and 2-3 months. This result hints at a possible functional effect of ascorbate to ameliorate MRI.

Key words: ascorbate, myocardial reperfusion injury, oxidative stress.

\section{Introduction}

Coronary heart disease is the most common cause of morbidity and mortality worldwide, and its prevalence is steadily increasing, account-

\author{
Corresponding author: \\ Dr. Ramón Rodrigo \\ Molecular and Clinical \\ Pharmacology Program \\ Institute of Biomedical \\ Sciences \\ Faculty of Medicine \\ University of Chile \\ Independencia 1027 \\ Casilla 70058 \\ Santiago 7, Chile \\ Phone: 56-2-29786126 \\ Fax: 56-2-29786126 \\ E-mail: rrodrigo@med.uchile.cl
}


ing for up to $12.8 \%$ of overall mortality [1]. During the last two decades, the advances of reperfusion therapies, such as primary coronary angioplasty (PCA), have significantly reduced the acute myocardial infarction (AMI)-related mortality burden and improved the clinical outcomes of patients [2]. Aside from the ischemic necrotic area, restoring oxygen flow paradoxically causes further cell death through rapid and massive myocardial necrosis along with less intense yet more prolonged apoptosis and autophagy processes. These events lead to the configuration of a phenomenon known as myocardial reperfusion injury (MRI), which likely accounts for up to $50 \%$ of the final infarct size [3] and also leads to different complications, such as reperfusion arrhythmias and myocardial stunning [4]. A growing body of evidence suggests that this damage could be the result of oxidative stress generated in the cardiac tissue and triggered by both oxygen reactive species (ROS) and nitrogen reactive species (RNS) [5]. During ischemia, intracellular ROS production increases due to uncoupling of the mitochondrial respiratory chain, consuming residual oxygen, and a decrease in antioxidant defenses [5], leading to oxidative damage, inflammatory responses and cellular death by apoptotic, necrotic and autophagic pathways [6]. During reperfusion, the sudden increase in oxygen supply to the endothelium, cardiomyocytes and other cell types amplifies the basal rate of ROS production several times, mainly superoxide anion $\left(\mathrm{O}_{2}^{-}\right)$, and reaches a peak during the first few minutes [7]. The main sources of ROS include uncoupled endothelial nitric oxide synthase (NOS), mitochondria, fatty acid oxidation, xanthine oxidase activity and mainly NADPH oxidase activity $[5,8]$. This activates pro-inflammatory pathways such as nuclear factor- $\mathrm{\kappa} B$, triggering inflammation and apoptosis signaling [9]. In addition, inducible NOS activity enhances nitric oxide (NO) production, which rapidly reacts with $\mathrm{O}_{2}^{-}$, generating the highly toxic RNS peroxynitrite, which enhances free radical damage [10].

Taking into account that ROS produces rapid and irreversible damage to myocardial tissue and other organs, it is plausible that antioxidants may counteract this damage [11-15]. Numerous attempts using pharmacological strategies have targeted oxidative stress to prevent this phenomenon, but none of them have succeeded, probably due to a lack of consideration of the basic pharmacokinetic and pharmacodynamic aspects of antioxidants [5]. Ascorbate has been one of the most widely used antioxidants for this purpose due to its ability to directly reduce ROS [11]. In addition to its ROS scavenger actions, it exerts a complex modulation of numerous enzymes involved in ROS production, endothelial dysfunction, platelet aggregation and smooth muscle cell tone
[16]. Ascorbate increases NO synthesis, reduces ROS formation and contributes to the regulation of vascular tone $[17,18]$.

Taking into account the above considerations, a rational therapy for preventing $M R I$ could involve the use of ascorbate prior to reperfusion to enhance the antioxidant potential of myocardial tissue [5]. Two key aspects have to be considered with regard to this issue. First, ascorbate should be administered before the onset of reperfusion. Second, ascorbate has to reach high enough levels to counteract the superoxide and NO reactions, occurring at a rate that is $10^{5}$-fold greater than that at which superoxide reacts with ascorbic acid [19]. Accordingly, $10 \mathrm{mmol} / \mathrm{l}$ ascorbate is needed to effectively achieve its cardioprotective properties. This concentration is only reachable by giving intravenous ascorbate, which has been proven to be completely safe [20].

The aim of this study was to test the hypothesis that an ascorbate-based supplementation protocol can ameliorate MRI associated with reperfusion following PCA.

\section{Material and methods}

The protocol consisted of a randomized, multicenter, double-blind, placebo-controlled trial (ISRCTN registry: 56034553) conducted in 99 either-sex patients who had a diagnosis of ST-segment elevation acute myocardial infarction (STEMI) and an indication for primary PCA (angina and an electrocardiogram with an STEMI that concerned more than two contiguous leads $>2 \mathrm{~mm}$ ). The presentation should have occurred within $12 \mathrm{~h}$ of the onset of symptoms. Only patients older than 18 years who experienced their first myocardial infarction were enrolled. The patient enrolment took place from February 2013 to November 2015. The cardiovascular units involved in the study included the University of Chile Clinical Hospital, San Juan de Dios Hospital, and San Borja Arriarán Clinical Hospital. The exclusion criteria included a history of renal or hepatic insufficiency, heart failure (NYHA III or IV), cardiogenic shock, post-primary PCA TIMI grade flow $<3$, any serious medical comorbidity that indicated a life expectancy $<6$ months, pregnancy, history of renal stones, and glucose 6-phosphate dehydrogenase deficiency. Other causes of exclusion during follow-up are shown in Figure 1.

\section{Study population}

The participants were randomized at the time of enrolment to placebo $(n=53)$ or ascorbate-treatment groups $(n=46)$. The randomization sequence was centrally generated, stored, and assigned by using online randomization software in random permuted blocks, each consisting of a box 


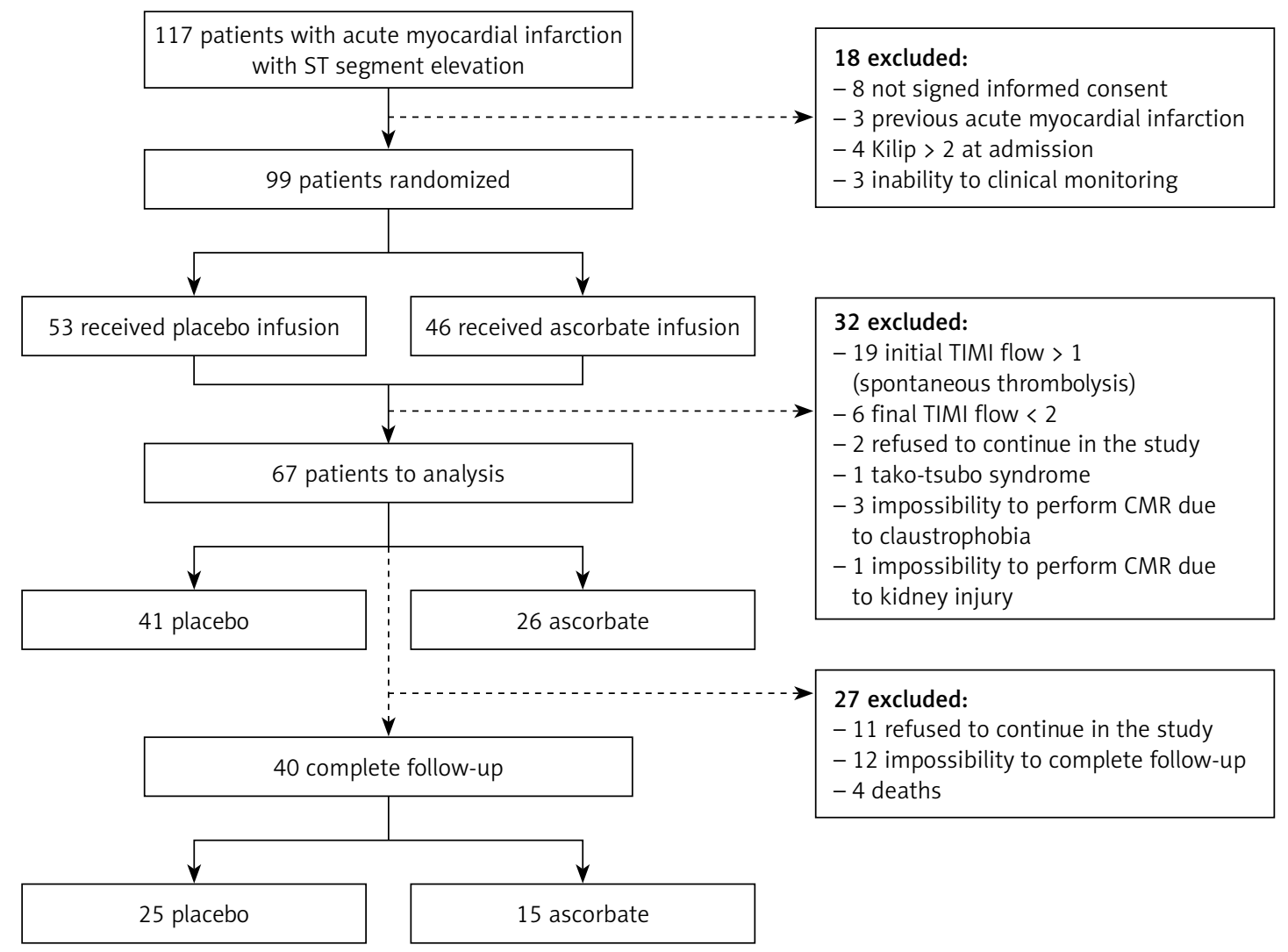

Figure 1. Disposition of patients. Randomization, treatment, and follow-up of participants

$C M R$ - cardiac magnetic resonance.

containing six treatment kits allocated in a placebo-to-vitamin treatment in a $1: 1$ ratio for each participating center. The treatment was initiated immediately after the randomization. For subjects allocated to the ascorbate-treatment group, the protocol began by administering an intravenous infusion of sodium ascorbate $(320 \mathrm{mmol} / \mathrm{l})$ at a flow rate of $10 \mathrm{ml} / \mathrm{min}$ during the initial hour and at $3 \mathrm{ml} / \mathrm{min}$ during the following $2 \mathrm{~h}$.

After the initial evaluation, the subjects in both groups were admitted to the catheterization laboratory in accordance with the standard protocol for AMI. An angiography was performed to identify the infarct-related artery, percentage of stenosis, and initial TIMI flow. Primary PCA was performed according to the current guidelines of each cardiovascular center. Catheterization was performed through the radial or femoral artery according to the clinician's decision. Then, the catheter was inserted into the artery and threaded to the coronary vessel. Once the occluded area was identified, balloon dilatation was carried out only in the culprit artery. The stent device implantation was decided according to need and the judgment of the physician in charge of the procedure. After the primary PCA, oral doses of ascorbate $(500 \mathrm{mg} / 12 \mathrm{~h})$ and $\alpha$-tocopherol (400 IU/day) were orally administered to the patients for 84 continuous days. For the subjects allocated to the placebo group, the oral dose of ascorbate was replaced with vegetable oil capsules (Procaps). The intravenous infusion of ascorbate was replaced with an equal volume of a sodium chloride solution having the same osmolality as the ascorbate infusion that was administered to patients in the ascorbate group. The pharmaceutical forms of ascorbate were indistinguishable from their respective placebo.

\section{Cardiac magnetic resonance assessment}

In 67 patients, cardiac magnetic resonance (CMR) assessments were performed 7-15 days after primary PCA (first CMR). In a subgroup of 40 patients, a second CMR was performed 2-3 months following primary PCA (second CMR). The magnetic resonance scanner specifications were as follows: Model: Siemens Sonata 1.5 tesla. Coil: 4-channel array coil. The imaging protocol included a cine balanced SSFP sequence and an inversion recovery PSIR sequence 10 min after the administration of $2 \mathrm{mmol} / \mathrm{kg}$ of intravenous gadolinium. Each sequence was performed in 7-mm slices in the short axis plane from the base to the apex and in the 2- and 4-chamber planes. Images were analyzed with the Argus software (Siemens, Germany) for the volumes and ejection fraction of 
the left ventricle, as well as with the segment software v1.9 (http://segment.heiberg.se) for quantification of the infarct size [21]. Both the determination and data obtained by these procedures were evaluated by 2 independent individuals who were blinded to the clinical data of the patients.

\section{Laboratory tests}

Immediately before the coronary artery occlusion was confirmed, a basal blood sample was drawn from the antecubital vein (M1). Three more blood samples were obtained at different times: immediately after reperfusion onset (M2), 6-8 h after balloon deflation (M3) and on hospital discharge (M4). Plasma samples were separated by blood centrifugation (3000 g for $10 \mathrm{~min}$ ). The red blood cell sediment was washed twice with saline and then subjected to hypotonic hemolysis with distilled water. The plasma and red blood cell lysate samples were stored at $-80^{\circ} \mathrm{C}$.

The plasma ascorbate concentration was determined by the fluorometric method [22]. The intracellular redox status in erythrocytes was assessed by measuring reduced glutathione (GSH) by fluorometry [23]. The antioxidant capacity of the plasma was assessed by the ferric reducing ability of plasma (FRAP) assay [24]. The plasma creatine kinase-MB (CK-MB) and total creatine kinase concentrations were measured using standard methods.

\section{Outcomes}

The primary outcome was defined as an infarct size assessment by CMR at 7-15 days. The secondary outcomes were an infarct size assessment by CMR at 2-3 months, left ventricular ejection fraction (LVEF) and indexed end-systolic volume (IESV) in both CMRs, antioxidant intracellular (GSH) and extracellular (FRAP) potential and plasma concentrations of creatine kinase-MB (CK-MB).

\section{Statistical analysis}

The Shapiro-Wilk test and distribution plots were used to test the normality of the distribution. The results were expressed as the mean \pm the standard deviation for the parametric variables and the median plus the interquartile range for the non- parametric variables. Significant differences for the normally distributed variables were compared by Student's $t$-test, whereas non-normally distributed variables were compared using the Mann-Whitney $U$ test. Comparisons of the categorical variables among the different groups of patients were performed by means of the $\chi^{2}$ test. The difference between the groups was considered significant with a $p$-value $<0.05$. All statistical analyses were performed using Stata version 10.1 (Stata Corporation) and Graph Pad Prism 5.0.

\section{Ethics}

All procedures performed in the study were in accordance with the ethical standards of the Institutional and/or National Research Committee and with the 1964 Helsinki Declaration and its later amendments or comparable ethical standards. Ethical approval of the study was obtained from the local Institutional Ethics Committees. Informed written consent was obtained from all participants before initiating any participation in the trial.

\section{Results}

The study population consisted of 99 patients with comparable baseline characteristics. Thirtytwo patients were excluded. Sixty-seven patients underwent a CMR assessment on days 7-15, and 40 patients completed the follow-up with a second CMR after 2-3 months (Figure 1). Four patients died during follow up, 3 from cardiogenic shock and 1 from nosocomial pneumonia. There were no adverse events that could be attributed to the antioxidant supplementation treatment (Table I).

\section{Clinical characteristics}

The baseline characteristics of the study population are presented in Table I. Angiographic characteristics were also registered (Table II). During a median follow-up of 3 months, the primary outcome of the study was analyzed in 40 (40.4\%) participants. Fifty-nine patients were excluded from the study (Figure 1).

\section{Cardiac resonance magnetic assessment}

There was no significant difference in infarct size between the groups either in the first or the second CMR (Table III).

The LVEF assessed by CMR and the IESV also showed no significant difference between groups in the first or second CMR (Table III). Additionally, to analyze the individual changes in the LVEF experienced by the patients in both groups throughout the follow-up, the difference between the LVEF obtained in the two CMRs after the PCA was calculated (Delta LVEF). The changes in the LVEF obtained with this method showed significantly higher values for the ascorbate group than for the placebo group $(p<0.05)$ (Figure 2$)$.

\section{Oxidative stress-related parameters and cardiac biomarkers}

The mean plasma ascorbate and FRAP levels were significantly higher in $M 2$ and $M 3$ in the ascorbate group compared to the placebo group $(p<0.05)$. The erythrocyte GSH levels showed no significant differences between the groups at M1 or M4. However, at M2 and M3, the GSH levels for 
Table I. Baseline characteristics of enrolled patients

\begin{tabular}{|c|c|c|c|c|}
\hline Variable & Placebo & Ascorbate & Total & $P$-value \\
\hline$N$ & 53 & 46 & 99 & \\
\hline Age [years] & $56.16 \pm 8.51$ & $59.2 \pm 11.98$ & $57.47 \pm 10.2$ & 0.7801 \\
\hline Systolic BP at admission [mm Hg] & $152.56 \pm 15.42$ & $147.89 \pm 43.01$ & $150.17 \pm 28.08$ & 0.4530 \\
\hline Diastolic BP at admission [mm Hg] & $87.10 \pm 6.57$ & $90.98 \pm 67.21$ & $89.25 \pm 15.08$ & 0.4580 \\
\hline Heart rate [beats/min] & $78.80 \pm 4.32$ & $80.11 \pm 24.01$ & $79.67 \pm 18.28$ & 0.6742 \\
\hline $\mathrm{BMI}\left[\mathrm{kg} / \mathrm{m}^{2}\right]$ & $29.2 \pm 4.52$ & $26.62 \pm 4.71$ & $28.00 \pm 4.70$ & 0.5512 \\
\hline Onset of symptoms - balloon angioplasty [h] & $5.27 \pm 2.78$ & $5.07 \pm 2.50$ & $5.20 \pm 2.63$ & 0.8102 \\
\hline Mortality 3 months, $n(\%)$ & $2(3.77)$ & $2(4.34)$ & $4(4.04)$ & 0.7123 \\
\hline Recurrence of AMI - 3 months, $n(\%)$ & $0(0)$ & $0(0)$ & $0(0)$ & - \\
\hline \multicolumn{5}{|l|}{ Comorbidities, $n(\%)$ : } \\
\hline Hypertension & $14(26.41)$ & $13(28.26)$ & $27(27.27)$ & 0.9050 \\
\hline Diabetes mellitus & $7(13.21)$ & $2(4.34)$ & $9(9.09)$ & 0.1231 \\
\hline Smoking & $16(30.19)$ & $15(32.61)$ & $31(31.31)$ & 0.2410 \\
\hline Dyslipidemia & $10(18.87)$ & $4(8.69)$ & $14(14.14)$ & 0.1982 \\
\hline \multicolumn{5}{|l|}{ Previous drug treatment, $n(\%)$ : } \\
\hline ACE inhibitor and/or ARBs & $7(13.21)$ & $6(13.04)$ & $13(13.13)$ & 0.2904 \\
\hline Aspirin & $4(7.54)$ & $4(8.69)$ & $8(8.08)$ & 0.5509 \\
\hline$\beta$-Blockers & $5(9.43)$ & $4(8.69)$ & $9(9.09)$ & 0.4464 \\
\hline Statins & $3(5.66)$ & $2(4.34)$ & $5(5.05)$ & 0.6098 \\
\hline Diuretics & $8(15.09)$ & $10(21.74)$ & $18(18.18)$ & 0.3659 \\
\hline Calcium channel blockers & $1(1.88)$ & $1(2.17)$ & $4(4.04)$ & 0.5233 \\
\hline Nitrates & $0(0.00)$ & $0(0.00)$ & $0(0.00)$ & - \\
\hline Fibrates & $1(1.88)$ & $0(0.00)$ & $1(1.01)$ & 0.2420 \\
\hline Aldosterone receptor antagonist & $1(1.88)$ & $0(0.00)$ & $1(1.01)$ & 0.2420 \\
\hline Antiarrhythmic & $0(0.00)$ & $0(0.00)$ & $0(0.00)$ & - \\
\hline Anticoagulants & $0(0.00)$ & $0(0.00)$ & $0(0.00)$ & - \\
\hline \multicolumn{5}{|l|}{ Post-follow-up drug treatment: } \\
\hline$N$ & 25 & 15 & 40 & \\
\hline ACE inhibitor and/or ARBs, $n(\%)$ & $25(100.00)$ & $15(100.00)$ & $40(100.00)$ & - \\
\hline Aspirin, $n(\%)$ & $25(100.00)$ & $15(100.00)$ & $40(100.00)$ & - \\
\hline$\beta$-Blockers, $n(\%)$ & $25(100.00)$ & $15(100.00)$ & $40(100.00)$ & - \\
\hline Statins, $n(\%)$ & $25(100.00)$ & $15(100.00)$ & $40(100.00)$ & - \\
\hline Diuretics, $n(\%)$ & $6(24.00)$ & $3(20.00)$ & $9(22.50)$ & 0.4190 \\
\hline Calcium channel blockers, $n(\%)$ & $2(8.00)$ & $1(6.66)$ & $3(7.50)$ & 0.4503 \\
\hline Aldosterone receptor antagonist, $n$ (\%) & $1(4.00)$ & $1(6.66)$ & $2(5.00)$ & 0.6129 \\
\hline Antiarrhythmic, $n$ (\%) & $0(0.00)$ & $0(0.00)$ & $0(0.00)$ & - \\
\hline Anticoagulants, $n(\%)$ & $0(0.00)$ & $0(0.00)$ & $0(0.00)$ & - \\
\hline
\end{tabular}

An intention-to-treat analysis was considered. Values are expressed as $n$ (\%) or mean \pm standard deviation. BP - blood pressure, $B M I$ - body mass index, AMI - acute myocardial infarction, ACE - angiotensin-converting enzyme, ARBs - angiotensin II receptor blockers, NS - non-significant. 
Table II. Angiographic characteristics of enrolled patients

\begin{tabular}{|c|c|c|c|c|}
\hline Variable & Placebo $(n=53)$ & Ascorbate $(n=46)$ & Total $(n=99)$ & $P$-value \\
\hline \multicolumn{5}{|l|}{ Compromised artery, $n(\%)$ : } \\
\hline Anterior descending artery & $27(50.94)$ & $24(50.00)$ & $51(51.51)$ & 0.2524 \\
\hline Right coronary artery & $21(39.62)$ & $12(26.09)$ & $33(33.33)$ & 0.2351 \\
\hline Circumflex artery & $5(9.43)$ & $10(21.74)$ & $15(15.15)$ & 0.5812 \\
\hline \multicolumn{5}{|l|}{ Surgical approach, $n(\%)$ : } \\
\hline Radial artery & $51(96.22)$ & $40(86.95)$ & $91(91.91)$ & 0.7910 \\
\hline Femoral artery & $2(3.77)$ & $6(14.04)$ & $8(8.08)$ & 0.7012 \\
\hline \multicolumn{5}{|l|}{ Initial TIMI flow, $n(\%)$ : } \\
\hline $0-1$ & $45(84.91)$ & 35 (76.09) & $80(80.80)$ & 0.5460 \\
\hline $2-3$ & $8(15.09)$ & $11(23.91)$ & $19(19.19)$ & 0.8124 \\
\hline \multicolumn{5}{|l|}{ Final TIMI flow, $n(\%)$ : } \\
\hline $0-1$ & $2(3.77)$ & $4(8.69)$ & $6(6.06)$ & 0.2452 \\
\hline $2-3$ & $51(96.23)$ & $42(91.31)$ & $93(93.93)$ & 0.3949 \\
\hline
\end{tabular}

An intention-to-treat analysis was considered. Values are expressed as $n$ (\%). NS - non-significant.

Table III. Cardiac resonance assessments

\begin{tabular}{|lccc|}
\hline Outcome & Placebo $(n=25)$ & Ascorbate $(n=15)$ & $P$-value \\
\hline \begin{tabular}{l} 
Infarct size $(\%):$ \\
\hline $7-15$ days
\end{tabular} & $21.5(17.0-34.2)$ & $17.0(13.0-36.0)$ & 0.6646 \\
\hline $2-3$ months & $19.0(14.0-34.0)$ & $21.0(14.0-34.0)$ & 0.9642 \\
\hline \begin{tabular}{l} 
LVEF $(\%):$ \\
\hline $7-15$ days
\end{tabular} & $49.1(41.0-59.4)$ & $47.3(40.0-56.4)$ & 0.5403 \\
\hline $2-3$ months & $47.5(38.0-61.7)$ & $54.6(39.9-64.8)$ & 0.4155 \\
\hline IESV [ml/m ${ }^{2}$ : & $45.0(28.2-53.4)$ & $46.9(25.0-54.5)$ & 0.8461 \\
\hline 7-15 days & $47.0(34.0-56.5)$ & $39.0(25.0-60.8)$ & 0.3890 \\
\hline
\end{tabular}

Values are expressed as median (interquartile range). LVEF - left ventricular ejection fraction, IESV - indexed end systolic volume.

the ascorbate group were half of the respective placebo values $(p<0.05)$. The levels of the myocardial damage biomarker CK-MB were not significantly different between the groups at baseline or 6-8 $\mathrm{h}$ after PCA (Table IV).

\section{Discussion}

This study presents a novel strategy for protecting the heart against reperfusion damage based on the administration of high doses of ascorbate to patients with $\mathrm{AMI}$ who are undergoing primary PCA. We previously presented preliminary data, demonstrating for the first time that high plasma ascorbate levels significantly improved microvascular dysfunction [25].

This study was based on the paradigm of cardioprotection resulting from the high antioxidant

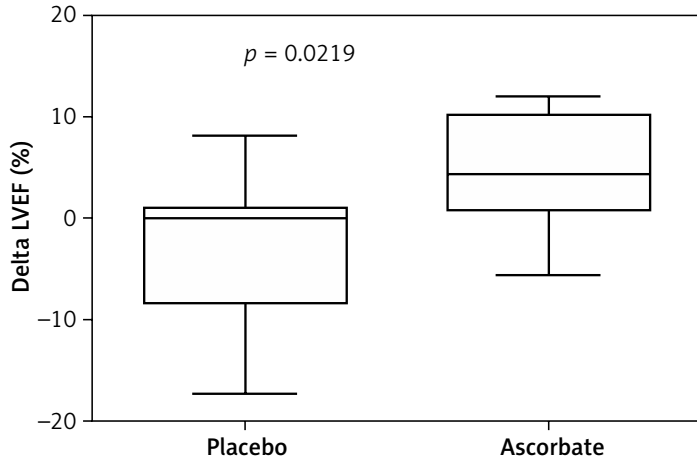

Figure 2. Difference between the LVEF obtained in the two CMRs after the PCA (Delta LVEF) ( $n$ placebo $=25 ; n$ ascorbate $=15$ ). Mann-Whitney $U$ test was applied. The delta LVEF obtained with this method showed significantly higher values for the ascorbate group than for the placebo group 


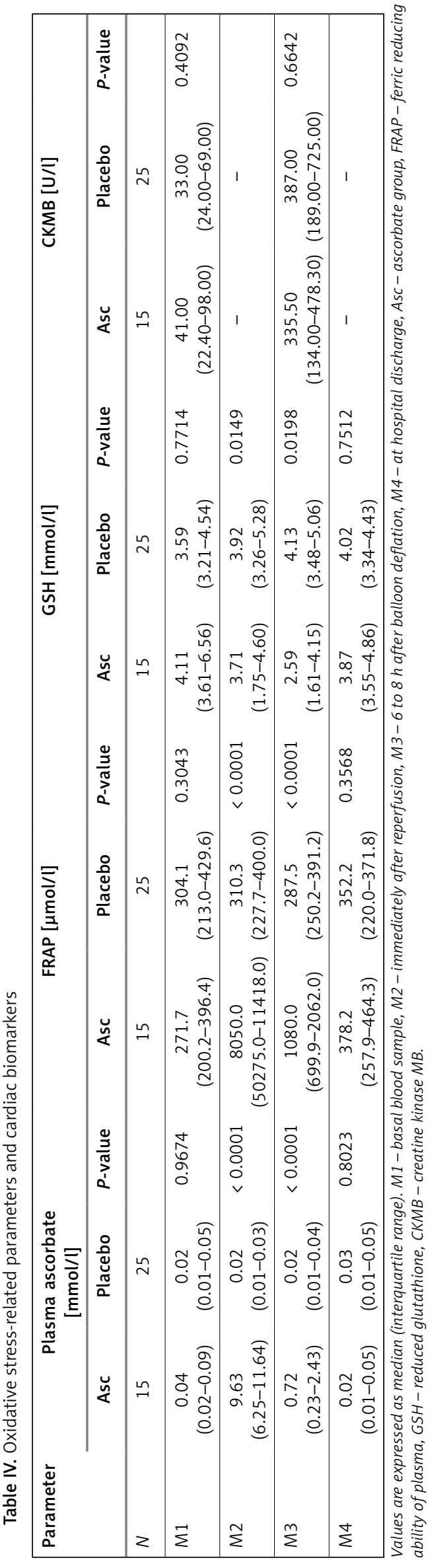

doses that we previously presented [11]. Ascorbate patients did not show a significant difference in infarct size, ejection fraction, or the prespecified CMR outcomes, compared to the placebo group. However, we found that ascorbate patients showed an improvement in change of ejection fraction determined with CMR between 7-15 days and 2-3 months. Although this parameter was not a prespecified one, it hints at a possible functional effect of our ascorbate protocol.

We attempted to minimize the deleterious effect of oxidative stress in ischemia-reperfusion injury to achieve a marked enhancement of the blood antioxidant potential at the onset of reperfusion. In fact, the higher plasma FRAP levels in the ascorbate group (Table IV) should involve an increased capacity to mitigate the oxidative damage associated with reperfusion. This favorable condition was maintained for at least $6-8 \mathrm{~h}$ following the onset of reperfusion, a phase characterized by the prevalence of a pro-oxidant state. However, high plasma ascorbate levels were accompanied by an unexpected decrease in the intracellular antioxidant capacity, as assessed by a decreased erythrocyte GSH (Table IV). A possible mechanistic explanation for this phenomenon is that GSH consumption is due to the recycling of ascorbate [26-28]. The decreased erythrocyte GSH levels could lead to increased vulnerability of cardiac tissue to oxidative damage. Nevertheless, there was no evidence of increased damage because supplemented patients did not have either a higher infarct size or increased cardiac injury serum biomarkers (Tables III and IV, respectively). Instead, patients in the ascorbate group achieved a functional benefit in the ejection fraction (Figure 2). Therefore, further studies are needed to determine the actual mechanism through which ascorbate influences the redox status in these patients.

The morphological and functional effects of ascorbate in this setting were assessed through CMR imaging, which is widely accepted as the gold standard for measuring infarct size in clinical practice [29-33]. In addition, CMR allows accurate and reproducible measurements of the ejection fraction, ventricular volumes and cardiac mass [29]. We chose the infarct size because it has been established as an important trial outcome by the Joint ESC/ACCF/AHA/WHF Task Force for the Redefinition of Myocardial Infarction [34]. Furthermore, infarct size is a validated assessment in trials that evaluate the amelioration of reperfusion injury [35-37] because its variation reflects the interaction of multiple physiologic and metabolic factors while providing a direct measure of the amount of myocardial cell loss [38].

Our results further suggest functional improvement of the ventricle induced by ascorbate. It is 
noteworthy that in the CMRs performed on days 7-15, the ejection fraction was similar in both groups, and the differences were not evident until 2-3 months following PCA. The mechanism whereby the present intervention ameliorated the persistent ejection fraction impairment remains to be elucidated. An analysis of these results should take into consideration the fact that previous studies performed using oral antioxidants alone following AMI showed no improvement in clinical outcomes [39]. Therefore, it may be plausible to assume that the high plasma ascorbate concentration that was achieved early during reperfusion played a key role in triggering this beneficial effect. It is likely that the FRAP elevation due to high plasma ascorbate levels at the onset of reperfusion are able to exert a prophylactic effect against the lethal reperfusion occurring during primary PCA [5]. To our knowledge, there are no previous studies using ascorbate doses that are capable of achieving the plasma concentrations reached in this study.

Previous attempts to prevent MRI through antioxidants showed disappointing results [40]. These studies included different types of antioxidants, including ascorbate, without clinical benefit [4145]. There are basic pharmacokinetic aspects of ascorbate that may explain why our intervention showed an effective clinical benefit based on the following considerations:

- Patients in the ascorbate group exhibited ascorbate levels high enough to block the reaction of superoxide with nitric oxide (>10 mmol/l) - levels that could not be reached by oral doses in the previous studies.

- Ascorbate was administered prior to the onset of reperfusion, when the MRI occurs. It should be taken into account that ROS burst and MRI occur immediately after the coronary blood flow restoration [7], and all the strategies aimed at preventing MRI should be applied prior to reperfusion [11].

The analysis of these results is subject to inherent limitations. First, at the moment of enrolment, many patients with a clinical diagnosis of AMI (angina and EKG classic characteristics) presented with an initial TIMI flow $>1$, independent of primary PCA. Furthermore, we could not document the exact time of onset of reperfusion. Clinical follow-up and laboratory tests, but not CMR, were performed in these patients. Second, despite using the most validated method to assess infarct size, the main limitation of our study was the absence of a method for determining the start point of myocardial damage (area at risk). This area depends on many factors, including a compromised artery, occluded segment, myocardial collateral irrigation, individual myocardial anato- my characteristics, and frequent variability in the anatomic distribution of the coronary arteries. Third, in a few cases, it was particularly difficult to assess the ischemia time due to the atypical presentations of AMI we well as the different pain threshold of some patients (e.g.: diabetics and older patients). Fourth, contrast enhanced CMR has absolute and relative contraindications, such as claustrophobia, renal failure, arrhythmia and severe dyspnea, which led to a significant drop in the number of patients who could participate in the primary outcome analyses (Figure 1).

In conclusion, the present study was designed to determine the cardioprotective effect of ascorbate in AMI patients undergoing primary PCA. The results support the view that a high intravenous dose administration prior to reperfusion onset followed by oral doses of ascorbate did not result in amelioration of infarct size or ejection fraction. However, we found that the ascorbate group showed an improvement in change of ejection fraction determined with CMR between 7-15 days and 2-3 months. Further studies are required to establish antioxidant protocols as adjuvant therapies during PCA to prevent MRI and improve the clinical outcomes of patients with AMI.

\section{Acknowledgments}

This research was supported by National Fund for Scientific and Technological Development (FONDECYT) grant number 1120594. The authors thank the technical assistance of Diego Soto, Juan Pinto, Shirley Rustom, Cristian Varas, Paulina Lecaros, Kjersti Nes, Pilar Varas and Lucio León.

\section{Conflict of interest}

The authors declare no conflict of interest.

\section{References}

1. Roe MT, Halabi AR, Mehta RH, et al. Documented traditional cardiovascular risk factors and mortality in nonST-segment elevation myocardial infarction. Am Heart J 2007; 153: 507-14.

2. Weaver WD, Simes RJ, Betriu A, et al. Comparison of primary coronary angioplasty and intravenous thrombolytic therapy for acute myocardial infarction a quantitative review. JAMA 1997; 278: 2093-8.

3. Yellon DM, Hausenloy DJ. Myocardial reperfusión injury. N Engl J Med 2007; 357: 1121-35.

4. Bolli R, Marbán E. Molecular and cellular mechanisms of myocardial stunning. Physiol Rev 1999; 79: 609-34.

5. Rodrigo R, Libuy M, Feliú F, Hasson D. Molecular basis of cardioprotective effect of antioxidant vitamins in myocardial infarction. Biomed Res Int 2013; 2013: 437613.

6. Hariharan N, Zhai P, Sadoshima J. Oxidative stress stimulates autophagic flux during ischemia/reperfusion. Antioxid Redox Signal 2011; 14: 2179-90.

7. Vanden Hoek TL, Li C, Shao Z, Schumacker PT, Becker LB. Significant levels of oxidants are generated by isolated 
cardiomyocites during ischemia prior to reperfusion J Mol Cell Cardiol 1997; 29: 2571-83.

8. Braunersreuther V, Montecucco F, Asrih M, et al. Role of NADPH oxidase isoforms NOX1, NOX2 and NOX4 in myocardial ischemia/reperfusion injury. J Mol Cell Cardiol 2013; 64: 99-107.

9. Chandrasekar B, Smith JB, Freeman GL. Ischemia-reperfusion of rat myocardium activates nuclear factor-kap$\mathrm{paB}$ and induces neutrophil infiltration via lipopolysaccharide-induced CXC chemokine. Circulation 2001; 103: 2296-302.

10. Wildhirt SM, Weismueller S, Schulze C, Conrad N, Kornberg A, Reichart B. Inducible nitric oxide synthase activation after ischemia/reperfusion contributes to myocardial dysfunction and extent of infarct size in rabbits: evidence for a late phase of nitric oxide-mediated reperfusion injury. Cardiovasc Res 1999; 43: 698-711.

11. Rodrigo R, Prieto JC, Castillo R. Cardioprotection against ischaemia/reperfusion by vitamins $C$ and $E$ plus $n-3$ fatty acids: molecular mechanisms and potential clinical applications. Clin Sci (Lond) 2013; 124: 1-15.

12. Dobutovic B, Sudar E, Teparcevic S, et al. Effects of ghrelin on protein expression of antioxidative enzymes and iNOS in the rat liver. Arch Med Sci 2014; 10: 806-16.

13. Khanavi M, Sabbagh-Bani-Azad M, Abdolghaffari AH, et al. On the benefit of galls of Quercus brantii Lindl. in murine colitis: the role of free gallic acid. Arch Med Sci 2014; 10: 1225-34.

14. Huang R, Zhong T, Wu H. Quercetin protects against lipopolysaccharide-induced acute lung injury in rats through suppression of inflammation and oxidative stress. Arch Med Sci 2015; 11: 427-32.

15. Tian M, Qing C, Niu Y, et al. Aminoguanidine cream ameliorates skin tissue microenvironment in diabetic rats. Arch Med Sci 2016; 12: 179-87.

16. Guney M, Oral B, Demirin H, Karahan N, Mungan T, Delibas N. Protective effects of vitamins $C$ and $E$ against endometrial damage and oxidative stress in fluoride intoxication. Clin Exp Pharmacol Physiol 2007; 34: 467-74.

17. Newaz MA, Yousefipour Z, Nawal NN. Modulation of nitric oxide synthase activity in brain, liver, and blood vessels of spontaneously hypertensive rats by ascorbic acid: protection from free radical injury. Clin Exp Hypertens 2005; 6: 497-508.

18. Wu F, Schuster DP, Tyml K, Wilson JX. Ascorbate inhibits NADPH oxidase subunit p47phox expression in microvascular endothelial cells. Free Radic Bio Med 2007; 42: 124-31.

19. Jackson TS, Xu A, Vita JA, Keaney JF Jr. Ascorbate prevents the interaction of superoxide and nitric oxide only at very high physiological concentrations. Circ Res 1998; 83: 916-22.

20. Padayatty SJ, Sun AY, Chen Q, Espey MG, Drisko J, Levine $M$. Vitamin C: intravenous use by complementary and alternative medicine practitioners and adverse effects. PLoS One 2010; 5: 11414

21. Helberg E, Engblom H, Engvall J, Hedström E, Ugander M, Arheden $\mathrm{H}$. Semi-automatic quantification of myocardial infarction from delayed contrast enhanced magnetic resonance imaging. Scand Cardiovasc J 2005; 39: 267-75.

22. Chung WY, Chung JK, Szeto YT, Tomlinson B, Benzie IF. Plasma ascorbic acid: measurement, stability and clinical utility revisited. Clin Biochem 2001; 34: 623-7.

23. Hissin PJ, Hilf R. A fluorometric method for determination of oxidized and reduced glutathione in tissues. Anal Biochem 1976; 74: 214-26.
24. Benzie IF, Strain JJ. The ferric reducing ability of plasma (FRAP) as a measure of "antioxidant power": the FRAP assay. Anal Biochem 1996; 239: 70-6.

25. Valls N, Gormaz JG, Aguayo R, et al. Amelioration of persistent left ventricular function impairment through increased plasma ascorbate levels following myocardial infarction. Redox Rep 2016; 21: 75-83.

26. Meister A. Glutathione-ascorbic acid antioxidant system in animals. J Biol Chem 1994; 2690: 9397-400.

27. Winkler BS. Unequivocal evidence in support of the nonenzymatic redox coupling between glutathione/glutathione disulfide and ascorbic acid/dehydroascorbic acid. Biochim Biophys Acta 1992; 1117: 287-90.

28. Winkler BS, Orselli SM, Rex TS. The redox couple between glutathione and ascorbic acid: a chemical and physiological perspective. Free Radic Biol Med 1994; 17: 333-49.

29. von Knobelsdorff-Brenkenhoff F, Schulz-Menger J. Cardiovascular magnetic resonance imaging in ischemic heart disease. J Magn Reson Imaging 2012; 36: 20-38.

30. Perazzolo Marra M, Lima JA, lliceto S. MRI in acute myocardial infarction. Eur Heart J 2011; 32: 284-93.

31. Kim HW, Farzaneh-Far A, Kim R. Cardiovascular magnetic resonance in patients with myocardial infarction: current and emerging applications. J Am Coll Cardiol 2009; 55: 1-16.

32. Martin TN, Groenning BA, Murray HM, et al. ST-segment deviation analysis of the admission 12-lead electrocardiogram as an aid to early diagnosis of acute myocardial infarction with a cardiac magnetic resonance imaging gold standard. J Am Coll Cardiol 2007; 50: 1021-8.

33. Desch S, Eitel I, de Waha S, et al. Cardiac magnetic resonance imaging parameters as surrogate endpoints in clinical trials of acute myocardial infarction. Trials 2011; 12: 204.

34. Thygesen K, Alpert JS, White HD. Joint ESC/ACCF/AHA/ WHF Task Force for the Redefinition of Myocardial Infarction. J Am Coll Cardiol 2012; 60: 1581-98.

35. Faxon DP, Gibbons RJ, Chronos NA, Gurbel PA, Sheehan F. The effect of blockade of the CD11/CD18 integrin receptor on infarct size in patients with acute myocardial infarction treated with direct angioplasty: the results of the HALTMI study. J Am Coll Cardiol 2002; 40: 1199-204.

36. Mahaffey KW, Granger CB, Nicolau JC, et al. Effect of pexelizumab, an anti-C5 complement antibody, as adjunctive therapy to fibrinolysis in acute myocardial infarction: the Complement inhibition in myocardial infarction treated with thrombolYtics (COMPLY) trial. Circulation 2003; 108: 1176-83.

37. Ross AM, Gibbons RJ, Stone GW, Kloner RA, Alexander RW. A randomized, double-blinded, placebo-controlled multicenter trial of adenosine as an adjunct to reperfusion in the treatment of acute myocardial infarction (AMISTAD-II). J Am Coll Cardiol 2005; 45: 1775-80.

38. Lowe JE, Reimer KA, Jennings RB. Experimental infarct size as a function of the amount of myocardium at risk. Am J Pathol 1978; 90: 363-79.

39. Yizhou Y, Jing L, Zhongxiang Y. Effect of antioxidant vitamin supplementation on cardiovascular outcomes: a meta-analysis of randomized controlled trials. PLoS One 2013; 8: e56803.

40. Braunersreuther $\mathrm{V}$, Jaquet $\mathrm{V}$. Reactive oxygen species in myocardial reperfusion injury: from physiopathology to therapeutic approaches. Curr Pharm Biotechnol 2012; 13: 97-114.

41. Singh RB, Wander GS, Rastogi A, et al. Randomized, double-blind placebo controlled trial of coenzyme Q10 
in patients with acute myocardial infarction. Cardiovasc Drugs Ther 1998; 12: 347-53.

42. Flaherty JT, Pitt B, Gruber JW, et al. Recombinant human superoxide dismutase (h-SOD) fails to improve recovery of ventricular function in patients undergoing coronary angioplasty for acute myocardial infarction. Circulation 1994; 89: 1982-91.

43. Dietary supplementation with $n-3$ polyunsaturated fatty acids and vitamin E after myocardial infarction: results of the GISSI-Prevenzione trial. Gruppo Italiano per lo Studio della Sopravvivenza nell'Infarto miocardico. Lancet 1999; 354: 447-55.

44. Yusuf S, Dagenais G, Pogue J, Bosch J, Sleight P. Vitamin E supplementation and cardiovascular events in high-risk patients. The Heart Outcomes Prevention Evaluation Study Investigators. N Engl J Med 2000; 342: 154-60.

45. Cung TT, Morel O, Cayla G, et al. Cyclosporine before $\mathrm{PCl}$ in patients with acute myocardial infarction. $\mathrm{N}$ Engl J Med 2015; 373: 1021-31. 